\section{El colegio invisible de Ángel Garma y el papel de sus colaboradores en el psicoanálisis argentino}

\section{The invisible college of Ángel Garma and the role of its collaborators in Argentine psychoanalysis}

Elena Quiñones Vidal

Catedrática del Departamento de Psicología Básica y Metodología/ Universidad de Murcia quinones@um.es

María Peñaranda Ortega

Profesora ayudante del Departamento de Psicología Básica y Metodología/Universidad de Murcia

$$
\text { mariap@um.es }
$$

\section{Elena García Quiñones}

Profesora del Departamento de Psicología Básica y Metodología/ Universidad de Murcia quinones@um.es

Universidad de Murcia. Facultad de Psicología Campus de Espinardo. Aptdo. de Correos 4021 30100 Espinardo - Murcia - España

Recibido para publicación en noviembre 2006. Aprobado para publicación en enero 2008.
QUIÑONES VIDAL, Elena;

PEÑARANDA ORTEGA, María; GARCÍA QUIÑONES, Elena. El colegio invisible de Ángel Garma y el papel de sus colaboradores en el psicoanálisis argentino. História, Ciências, Saúde Manguinhos, Rio de Janeiro, v.15, n.2, p.543-557, abr.-jun. 2008.

\section{Resumen}

La noción de que la esencia de la investigación científica es la producción de conocimiento y que la literatura científica constituye la manifestación de ese conocimiento hace que los acercamientos bibliométricos retraten a la ciencia por sus resultados. La identificación de estos grupos se realiza a través de las firmas conjuntas, agrupando a todos aquellos autores que mantienen relaciones directas o indirectas en función de las firmas de trabajos científicos. En el presente trabajo, los autores analizan la recepción del psicoanálisis y la evolución de las ideas psicológicas en Argentina, así como el papel jugado en el mismo por el español Ángel Garma, basándose en aspectos tanto biográficos como cuantitativos.

Palabras clave: psicoanálisis; Ángel Garma (1904-1993); historia de Argentina; colegio invisible.

\section{Abstract}

The idea that the essence of scientific investigation is the production of knowledge and that scientific literature constitutes a manifestation of this knowledge means that bibliometric approaches portray science through its results. These groups can be identified by their co-authorship, by grouping together all those authors who were directly or indirectly involved in writing the scientific papers. In this study, the authors analyze the reception of psychoanalysis and the development of ideas on psychology in Argentina, as well as the role played in this field by Spaniard Angel Garma, based on both biographical and quantitative data.

Keywords: psychoanalysis; Ángel Garma (1904-1993); history of Argentina; invisible college. 
$\mathrm{E}$ análisis bibliométrico es el fenómeno objetivable más importante en relación con la actividad científica (López Piñero, 1972). En todo lo concerniente a la ciencia, los indicadores bibliométricos son de uso obligado (Okubo, 1997; Araújo, Arencibia, 2002; López López, Tortosa Gil, 2002; Macedo, 2003). Mediante su empleo podemos analizar no sólo el crecimiento, el tamaño y la distribución de la literatura científica sino también la estructura social de los grupos que la producen y utilizan, el conocimiento de los procesos y la naturaleza de la ciencia con el fin de elaborar indicadores de actividad (López Piñero, 1972). Gracias a este análisis podemos obtener una radiografía de las teorías dominantes en un espacio de tiempo determinado y detectar sus grupos de investigadores que actúan como puntas de lanzas. La identificación de estos grupos se realiza a través de las firmas conjuntas (Price, Beaver, 1966; Peiró, 1981; Stack, 2002), agrupando a todos aquellos autores que mantienen relaciones directas o indirectas en función de las firmas de trabajos científicos. El método empleado para ello ha sido el de reunir en grupos a autores que firman juntos, abarcando tanto los trabajos que han realizado en cooperación entre todos ellos, como los firmados solo por parte del grupo o por algunos de los miembros en solitario (Brunn, O'Lear, 1999).

Para acceder a los hechos históricos de una manera más cuantitativa, nos apoyamos en la bibliometría como una de las técnicas más precisas en el estudio de los datos históricos (Pérez Matos, 2002). En el presente trabajo, los autores analizan la recepción del psicoanálisis y la evolución de las ideas psicológicas en Argentina, así como el papel jugado en el mismo por el español Ángel Garma, basándose en aspectos tanto biográficos - para referirse a sus trabajos en España - como cuantitativos. Así, se estudia el colegio invisible de Ángel Garma y se señalan algunas características del psicoanálisis en España y Latinoamérica a partir de su influencia.

Latinoamérica es actualmente uno de los tres grandes territorios psicoanalíticos existentes, junto con el europeo y el norteamericano (Zalbidea, Cantón, Carpintero, 1991). El 20\% de las organizaciones científicas que se reúnen en torno a la Asociación Psicoanalítica Internacional (IPA, en inglés) son latinoamericanas, con un centro neurálgico importante focalizado en Argentina, centrado en la Asociación Psicoanalítica Argentina (APA) y el Instituto de Psicoanálisis como entidad formativa, no sólo de psicoanalistas argentinos sino de todo el continente. Allí se formaron los primeros analistas de Porto Alegre (Mario Martins y Zaira de Bittencourt), Montevideo (Pérez Pastorini) o Rio de Janeiro (Alcyon Baer, María Perestrello y Oliveira).

Sin embargo, antes de que estas entidades se pusieran en marcha, algunos investigadores iberoamericanos se habían interesado por el psicoanálisis.

Según señala Ramón León (1984), en Latinoamérica, al contrario de lo que ocurrió en los Estados Unidos, ningún psicoanalista importante europeo visitó los países americanos de habla castellana o portuguesa en las dos primeras décadas del siglo XX. El primer psicoanalista hispanoamericano formado en Europa, Fernando Allende Navarro, volvió a Chile en 1925, año a partir del cual comenzó a difundirse en este país las ideas psicoanalíticas (Cesio, 1974). Debido a algunas características sociológicas y culturales - mayor influencia europea, fortalecimiento de ideas progresistas, menor presión de ideologías religiosas - fue en Argentina donde más arraigó el psicoanálisis (Arbiser, 2006). 
A la llegada, en la década de los 1930, los psicoanalistas europeos que huían de la Alemania nazi - María Langer que vivió en Montevideo y Ángel Garma en Argentina (Zalbidea, Cantón, Carpintero, 1991) - encontraron en Latinoamérica el terreno abonado. Al decir del propio Freud, fue el médico chileno Germán Greve quien primero se refirió al psicoanálisis en el Congreso Interamericano de Medicina e Higiene en Buenos Aires (1910). La ponencia "Sobre psicología y psicoterapia de ciertos estados angustiosos" fue recensionada por el mismo Freud en 1911, en Zentralblatt für Psychoanalyse. Pero los psicólogos latinoamericanos no volvieron a tener idea del decurso histórico del psicoanálisis hasta los escritos de Honorio Delgado, psiquiatra peruano, mencionado también por Freud en el Kurzer Abriss der Psychoanalyse, junto con la Revista de Psiquiatría y Disciplinas Conexas que Delgado y Valdirán fundaron en 1918.

Una idea de la importancia que van adquiriendo los psicoanalistas latinoamericanos hasta el establecimiento sistemático del mismo en el Cono Sur - con la llegada de Garma - la expone León en su estudio de 1982. En dicho trabajo se analiza el impacto de los escritos latinoamericanos en Europa, a través de la revista Intemationale Zeitschrift für Psychoanalyse, desde 1913 hasta su desaparición en 1941.

Antes, ningún otro autor latinoamericano logra la difusión de Honorio Delgado debido, como Abraham y Freud señalan, a la divulgación sistemática del psicoanálisis en Latinoamérica y a la minuciosa y precisa información que el autor facilita. Hasta 1927 se le sigue mencionando, fecha en que el propio Delgado se aleja del psicoanálisis. A pesar de que es en Brasil donde se funda la primera Asociación Psicoanalítica reconocida por la Asociación Psicoanalítica Internacional, el establecimiento y desarrollo institucional del psicoanálisis en Latinoamérica no se produce, como señalamos, hasta la llegada de Garma a Argentina (Quiñones Vidal, Vera Ferrándiz, 1988) y de Fromm a México (Arbiser, s.d.). Entonces el problema consistió como veremos en la falta de psicoanalistas entrenados.

En Buenos Aires, la labor realizada por Celes Cárcamo, Arnaldo Rascovsky y PichónRivière, durante la década de los 1930, había suministrado el abono necesario para que la inclusión en el grupo del propio Garma fructificara. Antes de formar una asociación, y para evitar lo ocurrido en Brasil, se dedicaron a formar a los futuros socios. Paralelamente en la APA se fundó un Instituto de Psicoanálisis Aplicado (Zak de Goldstein, 1974).

\section{Ángel Garma y el psicoanálisis}

En el caso de Garma, debemos destacar dos hechos en la conformación de su personalidad: (a) su estancia en la residencia de estudiantes que le pone en contacto con personalidades clave de la historia de España como por ejemplo García Lorca y Dalí; y (b) el conjunto de profesores que fueron responsables de su formación médica: Ramón y Cajal, Juan Negrín, Sanchís Banús, Lafora, Gregorio Marañón, Miguel Sacristán etc., resaltando en importancia la influencia de los dos últimos. Como él mismo dice, siguió con gran afán, no solamente sus enseñanzas médicas sino también las culturales e ideológicas. La conjunción de todas estas circunstancias determinó su carácter inquieto que posteriormente exhibió en todos aquellos lugares donde fue residiendo. Si bien es cierto que antes de graduarse ya acudía al manicomio que en Ciempozuelos dirigía el 
doctor Miguel Sacristán, uno de sus maestros. Las posibilidades que se le brindaron en su formación fueron aprovechadas por el joven médico, aun sin plantearse posibilidades en psicoterapia psicoanalítica.

Ángel Garma, siguiendo la tradición de los médicos españoles, decide completar su formación psiquiátrica en Alemania. Comenzó su estancia en Tübingen, trasladándose después a Berlín, a la Clínica Psiquiátrica del doctor Bonhoeffer; en esta ciudad entró en contacto con el Instituto de Psicoanálisis y decide comenzar su formación como psicoanalista. Conoce a la doctora Micaela Fabina, profesora de clínica, quien dirige su atención sobre el psicoanálisis. Con su descubrimiento, reconoce claramente el sentido de carencia que le afectaba y que algunos interpretan como decepción por la psiquiatría (Cesio, 1974) que le era incapaz de colmar y que le viene fundamentalmente de su relación con Marañón. Así lo indica en la entrevista que le hicimos en 1983: "También las ideas de Marañón me ayudaron a ese desarrollo en busca del conocimiento psicológico de los contenidos de los comportamientos humanos" (Garma, 1985, p.908).

En el Instituto Psicoanalítico de Berlín, dirigido por Eitingon - Garma fue el primero en acercarse al 'solitario' (citado por Carles Egea, 1982, p.57) - encuentra el centro más importante del momento. Se relaciona con grandes psicoanalistas como Franz Alexander, Theodor Benedek, Otto Fenichel, Karen Horney, Sandor Rado, Wilhelm Reich, Hanns Sachs y René Spitz, entre otros; es condiscípulo de Erich Fromm, y Thomas French. Conoció a Adler, Stekel y Jung. Se analizó con Theodor Reik, siendo su 'hermana' de análisis Paula Heimann. A los 27 años es nombrado miembro titular de la Asociación Psicoanalítica Alemana por el trabajo "La realidad y el ello en la esquizofrenia". Ese mismo año (1931) vuelve a España con la intención de trabajar en psicoanálisis.

Garma tenía muchas posibilidades de desarrollar institucionalmente el psicoanálisis en España. Él no pertenece, por su edad, a los grandes 'discípulos-apóstoles', difusores de la palabra viva del maestro en sus países de origen (como lo hicieron Eitingon y Jung en Suiza, Abraham en Alemania, Jones en Inglaterra, Ferenczi en Hungría), pero sí estaba en relación directa con muchos de ellos y pasó por el filtro de una formación analítica estándar común también a todos. Además, sus primeros trabajos fueron supervisados por el propio Freud quien, después de leerlos, le mandó una tarjeta - que Garma conservó en su biblioteca - en la que le agradece sus valiosos trabajos y le alentaba para seguir adelante, deseándole mucha suerte en su patria.

Una de las razones más poderosas para explicar la nula raigambre del psicoanálisis en forma de institucionalización en España debe buscarse, coincidiendo con Carles Egea (1982), en la inexistencia de relación de discipulaje y afiliación directa. Garma en 1931 estaba en disposición, por todas las características enumeradas más arriba, de terminar con esta situación en España y, con esta idea, se asentó de nuevo en nuestro país, significando la representación oficiosa del psicoanálisis.

\section{Entre España y Argentina}

Sabemos que las ideas de Freud fueron conocidas muy pronto en España y que desde la primera traducción en 1893 de "Los mecanismos psíquicos de los fenómenos histéricos", 
aparecido en la Gaceta Médica de Granada y en la Revista de Ciencias Médicas de Barcelona, los más importantes psiquiatras españoles de la época, Fernández Sanz, Rodríguez Lafora, Sacristán, Mira y López, Juarros, Sanchís Banús, se preocuparon por conocer las obras de Freud y de sus discípulos. En general, mantuvieron hacia las aportaciones psicoanalíticas la misma reserva escéptica que la mayoría de los psiquiatras de los distintos países europeos. No en vano, Freud saludó con especial entusiasmo la atención que sus ideas despertaban en el Burghölzli de Zurich, donde Bleuler y Jung trabajaban con enfermos neuróticos y sicóticos, desarrollando e investigando a partir de sus descubrimientos.

Sin embargo, la reserva inicial de la psiquiatría oficial española no impidió que las aportaciones psicoanalíticas conmovieran los cimientos organicistas de la psiquiatría y el estrecho campo de la conciencia, objeto del estudio de la psicología (Muñoz, 2001). Garma llega a Madrid en noviembre de 1931 y se marcha definitivamente en 1936, en vísperas de la Guerra Civil. En esos escasos cinco años desarrollará una intensa labor. En el trabajo de Quiñones Vidal y Muñoz Jiménez (1987), donde se analiza la actividad psicoanalítica en España desde 1923 a 1936, encontramos que es Garma el autor más productivo aunque publicando solamente desde 1930. Él solo reúne el 17,7\% de la producción total sobre psicoanálisis, siguiéndole Fernández Sanz con el 5,4\% y Rodríguez Lafora con el 5\%.

Comienza su práctica privada como psicoanalista centrándose sobre todo en el análisis didáctico de un futuro grupo de psicoanalistas, entre ellos los doctores Jerónimo Molina, José Solís y Mariano Bustamante, al mismo tiempo que desarrolla una importante actividad pública encaminada a dar a conocer el psicoanálisis y la importancia de una formación psicoanalítica para los psiquiatras. En este sentido colabora con los servicios de psiquiatría de Sanchís Banús y del doctor Sacristán. Participa en los cursos organizados por el doctor Rodríguez Lafora y en la cátedra de patología médica del doctor Marañón; finalmente obtiene una plaza de psiquiatría en el Tribunal de Menores y colabora asiduamente en la Revista Archivos de Neurobiología.

Durante estos años publica 19 artículos y el libro El psicoanálisis, la neurosis y la sociedad (1936). Theodor Reik, en el prólogo del libro, expresa claramente lo que fue durante estos años el objetivo fundamental de Garma:

\footnotetext{
Ha sentido usted la necesidad de hacer profundizar el conocimiento del psicoanálisis en la patria querida, de destruir confusiones que allí se habían creado y de demostrar a los colegas las posibilidades del método de psicología profunda. Yo no dudo que sus esfuerzos, nacidos de una rectitud interior y de una energía intelectual, le conducirán a usted a la meta deseada. Esté usted confiado y tranquilo, la verdad finalmente consigue triunfar.
}

La Guerra Civil española que estalló en julio de 1936 rompió estas expectativas. Garma que al estallar la guerra se encontraba en Francia, decide no regresar, quedándose en el país vecino hasta 1938. Allí comenzó a trabajar en el Instituto Psicoanalítico de París y a relacionarse con grandes psicoanalistas franceses como Laforgue o Lagache. Fue entonces cuando conoció a Celes Cárcamo, analista argentino que había ido para formarse con Spitz. En 1938, cuando se hace inminente la Segunda Guerra Mundial, decidirá emigrar a Argentina donde habían residido sus padres y nacido varios hermanos. En España los primeros años de la dictadura de Franco, terminada la Guerra Civil española (1936-1939), 
fueron dramáticos. En esos momentos no sólo el psicoanálisis sino la vida cultural y científica parecieron haberse extinguido. Tendremos que esperar a finales de la década de los 1940 para que vuelvan a surgir nuevos pioneros que en medio de un ambiente hostil e indiferente recuperen para el país el beneficio de las aportaciones psicoanalíticas.

Ya en 1943 Laín Entralgo dedica un amplio trabajo a la obra de Freud dentro de Estudios de historia de la medicina y de antropología médica, y en 1954, se inscribe en la Asociación Psicoanalítica Española presidida por Ramón del Portillo. Márquez (2005) llama la atención sobre la idea sostenida sobre la animadversión del franquismo hacia el psicoanálisis, llegando a la conclusión de que si esto hubiera sido así, nada hubiera impedido su erradicación.

Garma no pudo continuar su labor en España. Junto con Celes Cárcamo, A. Raskowsky, Pichón-Rivière, María Langer y G. Ferrari Hardoy, formarán la Asociación Psicoanalítica Argentina en Buenos Aires en 1938, iniciando contactos con Rascovsky y Pichón-Riviere, a quienes comenzó a psicoanalizar a partir de 1940. Los siguientes analizados fueron Simón Wencenblat y Arminda Aberastury. Como señalamos, en Francia había conocido a Celes Cárcamo por mediación del psiquiatra español Rof Carballo -médico argentino de origen español- con el que se encontró después en Buenos Aires y con el que compartió el trabajo didáctico.

Así, cuando Ángel Garma llega a Argentina ya había ganado bastante en experiencia acumulada en los años de España y Francia. En este país del nuevo continente encontró un ambiente muy propicio para el psicoanálisis que se había ido acondicionando en los medios médicos de Buenos Aires.

En Buenos Aires, la labor realizada por Celes Cárcamo, Arnaldo Rascovsky y Enrique Pichón-Rivière, durante toda la década de los 1930, había suministrado el abono necesario para germinar un psicoanálisis maduro como el conseguido por la APA. La incorporación de Garma al grupo dará el impulso decisivo para que la idea de un movimiento psicoanalítico argentino se pusiera en marcha. Una de sus primeras iniciativas fue la de sugerir que no se erigiera una asociación oficial hasta no contar con psicoanalistas bien formados.

El 15 de diciembre de 1942 se fundó la APA. El acta de fundación aparece firmada por seis miembros: Ángel Garma, Celes Cárcamo, Arnaldo Rascovsky, Enrique Pichón-Rivière, María Langer y Guillermo Ferrari Hardoy. El grupo inicial se oficializó en 1942. Todos comenzaron como miembros adherentes, condicionando su pertenencia a la presentación de un trabajo científico. Garma presentó el suyo un año después bajo el título "La realidad externa y los instintos en la esquizofrenia".

Los siguientes miembros del grupo fueron Luís Rascovsky, Enrique Racker, Luisa G. de Álvarez de Toledo, Alberto Tallaferro, Arminda Aberastury, E. E. Krapf, Matilde Rascovsky, Teodoro Schlossberg, Flora Scolnic y Simón Wencelblat. En sus reuniones no sólo se desarrollaban actividades profesionales, sino también una vida social activa (Guimón, 2005).

La necesidad de difundir el pensamiento psicoanalítico llevó a fundar la revista de la APA, cuyo primer número aparece en julio de 1943. En este mismo año, el resto de miembros fundadores también alcanzaron la categoría de titular y didactas de la Asociación. Garma sería el presidente de la misma, Cárcamo, su secretario y Rascovsky, director de publicaciones 
de lo que pasará a ser el órgano oficial de expresión de la Asociación: la Revista de Psicoanálisis de Argentina. En la publicación se pueden dibujar las líneas de actuación de los psicoanalistas en Argentina. Podemos decir que la política institucional de la APA seguiría por un lado una línea de adecuación a las instituciones médicas, encontrando en la medicina psicosomática y en el trabajo multidisciplinario un punto de encuentro. Por el otro, comienza la incorporación 'kleiniana' como sinónimo de psicoanálisis con lo cual se acomodaba a una perspectiva de consolidación del poder en la Asociación.

Con esta medida evitaron repetir el destino de la Sociedad Brasileira de Psicanálise primera en constituirse en América Latina, reconocida por la Asociación Psicoanalítica Internacional en 1929 - cuya desintegración fue inevitable y su revista, que apareció en 1928, sólo vio la luz por un número. Es preciso señalar que más de la mitad de las investigaciones sobre psicoanálisis y casi los $2 / 3$ de los análisis experimentales del comportamiento se concentran en la Universidad de Sao Paulo. Además, casi todo lo publicado sobre psicoanálisis pertenece a instituciones privadas - clínicas en su mayoría - mientras que sobre análisis se trabaja con instituciones públicas y actividades subvencionadas.

Por eso en Argentina, paralelamente a la APA, se fundó el Instituto de Psicoanálisis. De éste, Garma fue su primer director y también fue suyo el primer seminario que se celebró, centrado en el trabajo de Freud: Más allá del principio del placer.

En este Seminario, Garma incorpora los instintos de muerte a su teoría, abriendo el camino a nuevos descubrimientos, como, por ejemplo, los de M. Klein. "Así ... nos hizo posible a nosotros, sus alumnos, vencer las resistencias que se oponían a la aceptación de las últimas adquisiciones del psicoanálisis". Una vez más se desvela su carácter aperturista y renovador. Y, con este sello, se marcará la identidad del psicoanálisis argentino.

Como sabemos, el crecimiento institucional fue muy lento. A los psicoanalistas se les acumulaba el trabajo: realizaban cinco sesiones de análisis didáctico, seminarios, cursos, control de pacientes y un año de práctica psiquiátrica. Era evidente que la carrera no podía ser accesible para cualquier profesional, además hacían de analistas didácticos, analistas de control y también ejercían de profesores. Eso hicieron Garma, Arnaldo Rascovsky, PichónRivière, Langer y, a partir de 1945, Luis Rascovsky, llegando a contar con 104 profesionales (Ben Plotkin, 1996).

De Garma fue también la idea de organizar, en 1953, el primer symposium anual de la APA y también la de promover el primer congreso latinoamericano - 1956 - y el panamericano en 1964.

Respecto a su obra hay que decir que desde sus primeros trabajos Ángel Garma se limitó a realizar un psicoanálisis desde Freud con sus mismos presupuestos, pero llegando a conclusiones distintas y originales. Puede comprobarse en el trabajo con el cual consiguió la titularidad de la asociación alemana en 1931. En "La realidad y el ello en la esquizofrenia" plantea una concepción diferente sobre sicopatología a la que prevalecía con Freud en plena vigencia. También en sus dos primeros trabajos presentados en la APA: "La realidad exterior y los instintos en la esquizofrenia" y "La génesis del juicio de realidad: una teoría general de la alucinación", se conduce igual, con gran coherencia interna, dándole la vuelta a las conclusiones pero desde las mismas construcciones teóricas de Freud. Es esta originalidad y frescura, mantenida hasta sus últimas colaboraciones - que contienen la 
esencia del descubrimiento presto a ser compartido - lo que constituirá la más admirada cualidad por sus discípulos. De ello trataremos en el siguiente epígrafe.

\section{El colegio invisible de Ángel Garma}

Es verdad que sin el apoyo de sus discípulos y colaboradores, el papel jugado por Ángel Garma en Hispanoamérica hubiera sido otro. El Instituto de Psicoanálisis poco habría podido hacer sin la APA o la formación didáctica ofertada a los jóvenes psicoanalistas. Y tampoco sin la labor de todos aquellos que hicieron posible su incursión en la psicología universitaria y su expansión por todo el continente. Por ello se hace necesaria una investigación a fondo de quienes y cuantos lo hicieron posible. Para este fin utilizaremos el análisis bibliométrico de su producción científica y, concretamente, los instrumentos que nos permiten estudiar su productividad y su relación con sus colaboradores.

El análisis bibliométrico es el fenómeno objetivable más importante en relación con la actividad científica (López Piñero, 1972). En todo lo concerniente a la ciencia, los indicadores bibliométricos son de uso obligado (Okubo, 1997; Araújo, Arencibia, 2002; López, Tortosa, 2002; Macedo, 2003). Mediante su empleo podemos analizar no sólo el crecimiento, el tamaño y la distribución de la literatura científica sino también la estructura social de los grupos que la producen y utilizan, el conocimiento de los procesos y la naturaleza de la ciencia con el fin de elaborar indicadores de actividad (López Piñero, 1972). Para acceder a los hechos históricos de una manera más cuantitativa, nos apoyamos pues en la bibliometría como una de las técnicas más precisas en el estudio de los datos históricos (Pérez Matos, 2002).

La noción de que la esencia de la investigación científica es la producción de conocimiento y que la literatura científica constituye la manifestación de ese conocimiento (Carpintero y Peiró, 1983) hace que los acercamientos bibliométricos retraten a la ciencia por sus resultados.

Publicar los trabajos y los resultados de las investigaciones es la actividad suprema de los científicos, por ello ha sido extensamente contemplada por los analistas de la ciencia (Prpic, 1996, 2002; Basu, 1999; Melin, 2000 ). Según Price, un científico es "any person who has ever published a scientific paper" (Price, 1973). "Whenever a man labors, produces something new and the result is a publication, then he has been doing what I call Science" (Price, 1969). Publicar los resultados de sus investigaciones es una obligación que satisface a los científicos (Merton, 1957).

Los nuevos conocimientos puestos al día por los investigadores tienen que ser transformados en información disponible para la comunidad científica (Carpintero, Peiró, 1983). Los científicos no sólo deben exponer sus trabajos en forma de conocimiento público sino también han de tener acceso a los trabajos proporcionados por el resto de colegas. La investigación se realiza en un contexto de intercambio.

Así pues, el hecho de que el sistema de la publicación científica haya sobrevivido en la ciencia moderna es debido, paradójicamente, al deseo de los científicos de proteger su propiedad intelectual. El nuevo conocimiento científico es la creación personal de un investigador y la demanda sobre su descubrimiento únicamente se puede realizar a través de la publicación (Merton, 1957). 
El sistema de recompensas, basado en el reconocimiento del trabajo, subraya la importancia de la propia publicación: la única manera de dispersar los resultados de la investigación por el mundo es publicándolos. La publicación tiene, por tanto, tres objetivos: propagar los hallazgos científicos, proteger la propiedad intelectual y ganar fama (Okubo, 1997). Los científicos están comprometidos en publicar su trabajo y la publicación justifica su existencia (Soon, 1996).

Los aspectos a estudiar en un análisis bibliométrico son fundamentalmente cuatro, según López López (1996): productividad, materias, citas y colaboración. Siguiendo esta pauta general, obtendremos los resultados que necesitamos para nuestra investigación, por medio de estos indicadores bibliométricos.

Como sabemos, un indicador es un parámetro que se utiliza para evaluar cualquier actividad. En el caso de los indicadores bibliométricos, nos sirven para medir los avatares de la literatura científica (Sancho, 1990; Araújo, Arencibia, 2002). Estos parámetros son la medida indirecta de la comunidad científica, su estructura y sus resultados (Okubo, 1997). A través de la coautoría de las publicaciones es posible también analizar el grado de colaboración de los autores (Carpintero, Peiró, 1983; Alcaín, Gálvez, 1998). La tendencia general es el aumento de publicaciones de varios autores en revistas, aunque el porcentaje para ciencias sociales es menor que en ciencia y tecnología (Peiró, 1981; Civera; Martínez, 1995; López López, 1996; López Piñero, Terrada, 1992; López López, Tortosa, 2002).

El grado de colaboración también es relevante para la productividad. De hecho, uno de los desarrollos más importantes para la cooperación es su relación directa con la misma (Hollis, 2001). Los autores más productivos son aquellos que más colaboran (Civera, 1994). La autoría múltiple se ha incrementado mucho en las últimas décadas (Zuckerman, 1977), aunque como dijimos, hay que tener en cuenta que el grado de colaboración varía de unas disciplinas a otras y entre ramas de una misma disciplina (Stack, 2002).

La ciencia actual se caracteriza por una clara tendencia a la autoría múltiple (Melin, 2000), con un promedio de 2,5 firmas por trabajo (López Piñero, 1972). Según Price, el porcentaje de trabajos realizados por un solo autor ascendía a un $80 \%$ de los mismos, mientras que la autoría múltiple la conformaba el restante $20 \%$. En la big science o ciencia actual, el porcentaje se ha invertido claramente, con un $20 \%$ de artículos firmados por un único autor y un $80 \%$ firmados por dos o más autores.

Así, se supone que la ciencia moderna es el resultado del trabajo conjunto de los miembros de los equipos de investigación en determinadas áreas de conocimiento (Hollis, 2001). La envergadura de los problemas tratados en la ciencia experimental requiere el esfuerzo conjunto de todos los investigadores en un proyecto. Por otra parte, la ciencia presenta cierta tendencia a cristalizar, de tal manera que las grandes disciplinas y los grandes grupos de investigación absorben gran cantidad de recursos económicos y humanos. Por tanto, una de las principales aplicaciones de la bibliometría es el análisis de colaboración con el objetivo de detectar los grupos de autores que colaboran entre sí en un período temporal o en un área científica dada (Hollis, 2001; Licea de Arenas, Santillán-Rivero, 2002).

Gracias a este análisis podemos obtener una radiografía de las teorías dominantes en un espacio de tiempo y detectar sus grupos de investigadores que actúan como puntas de lanzas. La identificación de estos grupos se realiza a través de las firmas conjuntas (Price, 
Beaver, 1966; Peiró, 1981; Stack, 2002), agrupando a todos aquellos autores que mantienen relaciones directas o indirectas en función de las firmas de trabajos científicos. El método empleado para ello ha sido el de reunir en grupos a autores que firman juntos, abarcando tanto los trabajos que han realizado en cooperación entre todos ellos como los firmados solo por parte del grupo o por algunos de los miembros en solitario (Brunn, O'Lear, 1999).

De esta forma, los autores de la revista pueden ser clasificados en función del número de autores con los que colaboran directa o indirectamente y que constituyen un grupo: desde los que firman siempre en solitario, hasta aquellos vinculados a varios autores. Este es el procedimiento que hemos usado para representar la unión entre distintos autores.

Estos clusters o agrupamientos de científicos en forma de colegios invisibles representarían la infraestructura intelectual y social de la ciencia, ofreciendo información sobre un campo o especialidad científica, sobre el estado de la cuestión y del desarrollo actual e histórico de las ciencias con más eficacia que las fronteras disciplinares tradicionales (Sancho, 1990; Lutman, 1992; Shapiro, 1992; Alcaín, San Millán, 1993; Vinkler, 2000). La unidad de análisis de partida sería la firma conjunta que produce una relación entre varios autores, conformando un colegio invisible. Consideramos cabeza de un grupo al autor que ostenta mayor número de trabajos en colaboración, y por tanto, aglutina en torno a sí a los restantes (Price, Beaver, 1966; Peiró, 1981; Stack, 2002).

Sin embargo, Price reflejó un concepto de colegio invisible rígido y estático: "En conjunto, un científico puede relacionarse con un grupo de colegas que tiene un tamaño efectivo de unos pocos centenares de miembros; con seguridad, no puede relacionarse con 10.000" (Price, 1973).

Un manera de analizar el estudio de la cofirmación son los procedimientos de tipo gráfico (Civera, Martínez, 1995), por los que la globalidad de los autores quedaría instaurada en un gráfico que mostraría diferentes características seleccionadas para su estudio. La mayor parte de las críticas recibidas a este tipo de análisis se deriva de limitaciones sobre diseño del gráfico. No se podrían estudiar colegios invisibles extensos, con relaciones complejas entre los diferentes autores, o carecería de cualidades que permita comparaciones precisas (Civera, Martínez, 1995; Melin, Persson, 1998).

La utilidad del estudio de un colegio invisible es relatada por Peñaranda Ortega (2004):

La utilidad de este tipo de análisis no se limita a reseñar quiénes son los miembros que componen uno de esos colegios. Sirve también para detectar qué figuras son las que lideran el grupo y lo cohesionan, o analizar la productividad y eminencia de los grupos en comparación con la del investigador solitario. Plantean, pues, problemas de interés relacionados con la estructura y dinámica del funcionamiento científico, si bien los análisis han de ser enmarcados en un enfoque teórico desde el cual interpretarlos.

Según Peiró (1981), los indicadores más importantes de la colaboración son los contactos directos, las colaboraciones publicadas en artículos firmados juntos, las relaciones maestrodiscípulo y la influencia reconocida a través de trabajos publicados por medio de citas bibliográficas. Uno de los indicadores más utilizados para esta realidad social es el análisis de los colegios invisibles (Brunn, O'Lear, 1999).

Ya Price (1969) sugirió que los colegios invisibles estaban caracterizados por relaciones formales de científicos que mantenían relaciones informales de contacto, especialmente 
las distintas a los medios impresos. En medio de esta dinámica, surgían grupos estrechos de colaboradores que tendían a influirse entre sí (Brunn, O’Lear, 1999; Hollis, 2001). Este hecho es de especial relevancia para los historiadores quienes se dedicaron a estudiar estos grupos de influencia. Dos tendencias se manifestaron en este sentido. La propuesta de Crane (1972) y su análisis de la colaboración desde un punto de vista sociométrico y la concepción de Price y Beaver (1966) de colegio invisible como el estudio de la autoría conjunta por medio de las firmas realizadas en las publicaciones científicas.

De esta forma, se comprueba que la ciencia no es más que la mera unión de ramas científicas más alejadas o separadas entre sí, pero englobadas al fin y al cabo en el mismo plano (Tvire, Erno, 2001; Barabási et al., 2002). Desde este punto de vista, los autores investigan y firman los trabajos juntos, estableciendo una estrecha relación entre sí, y quedan vinculados con otros autores que, no habiendo publicado directamente con ellos, sí lo han hecho con sus colaboradores más directos.

Esta red de relaciones sociales establece grupos coherentes y permanentes de trabajo, con intereses y finalidades bien definidas, tanto teórica como metodológicamente (Carpintero, Peiró, 1981). Según Vivas y Urquijo (1999), este análisis de redes se adapta muy eficazmente al estudio de las publicaciones y sus interrelaciones.

\section{El impacto de la obra de Ángel Garma}

El impacto científico de Garma ha sido estudiado por Zalbidea, Cantón y Carpintero (1991) y Quiñones Vidal y Vera Ferrándiz (1988). Este impacto se produce principalmente por su obra Psicoanálisis de los sueños escrita en 1940. El alcance de su obra en el Social Sciences Citation Index y Science Citation Index se refleja en que 33 de sus trabajos son citados a lo largo del tiempo.

Respecto al trabajo de colaboración que presentamos, tenemos que reseñar que va referido a su producción en la Revista de Psicoanálisis que, como sabemos, se convirtió desde su inicio en el órgano de expresión de la APA y es en este contexto en el que tienen sentido nuestras afirmaciones al respecto.

Garma publica un total de sesienta trabajos en la Revista de Psicoanálisis, obteniendo así un nutrido grupo de colaboraciones derivadas de su relación con sus discípulos. El colegio invisible se obtiene del estudio de las publicaciones del autor con sus respectivos cofirmantes (Peñaranda Ortega, 2004).

Sus colaboradores directos son cuatro y uno de ellos es su mujer, confirmando el arraigo que desde el principio supuso la labor de los consortes de los pioneros del psicoanálisis argentino. Aunque Garma trabajó con 21 colaboradores distintos a lo largo de su vida productiva en la revista, su relación se materializa a través de estos cuatro. Comprobamos que de los 21 colaboradores que presentan sus trabajos, los más activos en la publicación conjunta son Elisabeth Goode de Garma y Elfriede Susana Lustig de Ferrer (que publican con Garma siete de sus 14 y 15 trabajos respectivos en la revista), y Alejandro Arbiser y Jorge O. Vinocur, con cinco trabajos cada uno.

Esta red primaria de colaboración, se amplía si consideramos los colaboradores que a su vez trabajan con estos asistentes directos. De estos 21 colaboradores directos, se desprenden 
un total de 86 colaboradores cercanos, lo cual le proporciona un índice de colaboración de 2,01, claro indicativo de un colegio invisible muy productivo y con una gran carga de miembros activos.

\section{Los discípulos españoles de Ángel Garma}

Los primeros discípulos de Garma en España fueron los médicos Portillo y Molina. Ambos conocían a Garma desde su estancia en Madrid. De hecho este último se había analizado con él. Ramón del Portillo, iniciador del grupo madrileño, llegará al psicoanálisis a través de sus lecturas personales y su contacto con Jerónimo Molina. En 1948 contactan con Garma para que les introduzca en la IPA a través del doctor Muller-Braunschweig y Margarita Steinback, una psicoanalista didacta con la que se analiza en Berlín hasta 1950. Ambos serán, junto con Portillo, el embrión del grupo madrileño de psicoanálisis.

Este selecto grupo de discípulos de Garma representarían otra forma de configuración de colegio invisible, puesto que ninguno colabora directamente con Garma, aunque todos admiten relaciones de discipulaje al estilo tradicional, donde más que de colaboración podemos hablar de influencias.

El doctor Garma visita España en 1952. Los integrantes del grupo, Ramón del Portillo, Juan Rof Carballo, Maria L. Herreros, Maria Teresa Ruiz, etc., habían iniciado las gestiones para ser reconocidos por el Estado, lo que a juicio de los fundadores redundaría en beneficio de la introducción del psicoanálisis en España y de un mayor desarrollo científico, facilitando el ejercicio de actividades docentes (seminarios, cursos, conferencias), al mismo tiempo que comunicaban su existencia y pedían el reconocimiento de la Asociación Internacional de Psicoanálisis (Muñoz, 2001).

En 1954 muere Margarita Steinback y Portillo visita Buenos Aires y varios componentes del grupo madrileño se psicoanalizan allí. Ramón del Portillo se analiza con Ángel Garma, Maria Teresa Ruiz con León Grinberg y Eduardo Blaise con Pichón-Rivière. A su vez viajan a Madrid Raskowsky, Cesio, Liberman, y Pola. En Barcelona, en 1955, se celebra el $1^{\circ}$ Congreso Iberoamericano de intercambio médico-psicológico en colaboración con la Asociación Psicoanalítica Argentina y en el que participan, entre otros, Ángel Garma, Obadía, David Liberman, Arnaldo y Matilde Raskowsky, García Vega, Rebeca Grinberg, Jorge Mom, Salomon Resnick, Edgardo H. Rolla, Alberto Tallaferro, Fidias Cesio, Diego García Reinoso, etc.

Paralelamente en estos mismos años, los doctores Bofill, Folch, Corominas, Abelló y Eskelinen de Barcelona y Rallo, Zamora y Pertejo de Madrid, que realizaban su formación en las Sociedades Psicoanalíticas de Suiza y París, habían iniciado las gestiones, junto con los doctores Luzes y Alvin de Portugal, para ser reconocidos como Sociedad Luso-Española de Psicoanálisis por la Sociedad Internacional de Psicoanálisis. El reconocimiento por la IPA se da en 1959. En 1990 visita España por última vez, recibiendo la medalla al Mérito Civil, logrando un reconocimiento que el Estado no había dado a los propios psicoanalistas. 


\section{REFERENCIAS}

ALCAÍN, María Dolores; GÁLVEZ, María. Evolución de las revistas españolas de psicología. Papeles del Psicólogo, Madrid, v.70, p.35-42. 1998.

ALCAÍN, María Dolores; SAN MILLÁN, María José.

Uso y tendencias de las técnicas bibliométricas en ciencias sociales y humanas a nivel internacional. Revista Española de Documentación Científica, Madrid, v.16, n.1, p.30-41. 1993.

ARAÚJO, Juan Antonio; ARENCIBIA, Ricardo. Informetría, bibliometría y cienciometría: aspectos teórico-prácticos. ACIMED, La Habana, v.10, n.4, p.165-170. 2002.

ARBISER, Sara.

Una historia del psicoanálisis en la Argentina. 2000. Disponible en: http://www.psiconet.com/ argentina/historia/textos.htm. Acceso en: 23 jun. 2006. s.d.

BASU, Aparna.

Science publication indicators for India: questions of interpretation. Scientometrics, New York, v.44, p.347-360. 1999.

BARABÁSI, Albert Laszlo et al.

Evolution of the social network of scientific collaborations. Physica A: Statistical Mechanics and its Applications, v.311, n.3-4, p.590-614. 2002.

BEN PLOTKIN, Mario.

Freud en la Universidad de Buenos Aires: la primera etapa hasta la creación de la carrera de Psicología. Estudios Interdisciplinarios de América Latina y el Caribe, v.7, n.1, p.26-32. 1996.

BRUNN, Stanley D.; O'LEAR, Shannon R. Research and communication in the 'invisible college' of the human dimensions of global change: the geography of latterday phenomenon. Global Environmental Change, Oxford, v.9, n.4, p.285-301. 1999.

CARLES EGEA, Francisco. La introducción del psicoanálisis en España (18931922). Tesis (Doctoral) - Facultad de Medicina, Universidad de Murcia, Murcia. 1982.

CARPINTERO, Helio; PEIRÓ, Jose María. The significance of the bibliometric methodology to the studies of the history of psychology. Revista de Historia de la Psicología, Valencia, v.4, n.1, p.21-32. 1983.

CARPINTERO, Helio; PEIRÓ, Jose María. Psicología contemporánea: teoría y métodos cuantitativos para el estudio de su literatura científica. Valencia: Editorial Alfaplús. 1981.
CESIO, Fidias.

Homenaje a Ángel Garma en ocasión de cumplir 70 años. Revista de Psicoanálisis, Buenos Aires, n.31, p.839-842. 1974.

CIVERA, Cristina.

La influencia de la psicología alemana en el desarrollo de la psicología española actual: el caso de J.C. Brengelmann y la terapia de conducta. Tesis (Doctorado) - Universidad de Valencia, Valencia. 1994.

CIVERA, Cristina; MARTÍNEZ, Tomás.

Aplicación de un método de análisis para la colaboración científica, a la obra científica de J.C. Brengelmann. Revista de Historia de la Psicología, Valencia, v.16, n.1-2, p.263-281. 1995.

CRANE, Diana.

Invisible colleges: disffusion of knowledge in scientific communities. Chicago: The University of Chicago Press. 1972.

GARMA, Ángel.

Entrevista a Ángel Garma. Murcia:

Departamento de Metodología y Análisis del Comportamiento/Universidad de Murcia. Documento no publicado. 1985.

GARMA, Ángel.

El psicoanálisis, la neurosis y la sociedad. Madrid: Ediciones de Archivos de Neurobiología. 1936.

GUIMÓN, Jesús Á.

Garma: otro fundador vasco. Avances en Salud Mental, v.4, n.2, p.1-7. 2005.

HOLLIS, Aidan.

Co-authorship and the output of academic economists. Labour Economics, v.8, n.4, p.503-530. 2001.

LEÓN, Ramón.

Una nota acerca de Honorio Delgado y su rol precursor en la historiografía sudamericana de la psicología. Revista de Historia de la Psicología, Valencia, v.5, n.1-2, p. 215-220. 1984.

LICEA DE ARENAS, Judith; SANTILLÁNRIVERO, Emma.

Bibliometría: ¿para qué?. Biblioteca Universitaria Nueva Época, México D.F., v.5, p.13-10. 2002.

LÓPEZ LÓPEZ, Pedro; TORTOSA GIL, Francisco. Los métodos bibliométricos en psicología. In: Tortosa, Francisco; Civera, Cristina (Coord.). Nuevas tecnologías de la información y documentación en psicología. Barcelona: Editorial Ariel. p.199-226. 2002.

LÓPEZ LÓPEZ, Pedro.

Introducción a la bibliometría. Valencia: Promolibro. 1996. 
LÓPEZ PIÑERO, Jose María.

El análisis estadístico y sociométrico de la literatura científica. Valencia: Centro de Documentación e Informática Médica/ Facultad de Medicina. 1972.

\section{LÓPEZ PIÑERO, Jose María; TERRADA, María} Luisa.

Los indicadores bibliométricos y la evaluación de la actividad médico-científica, 1: usos y abusos de la bibliometría. Medicina Clínica, Barcelona, v.98, n.2, p.64-68. 1992.

LUTMAN, Mark.

Bibliometric analysis as a measure of scientific output. British Journal of Audiology, London, v.26, n.6, p.323-324. 1992.

MACEDO, Raimundo Nonato Macedo dos. Produção científica: por que medir? o que medir? Revista Digital de Biblioteconomia e Ciência da Informação, v.1, n.1, p.22-38. 2003.

MÁRQUEZ, Isabel.

El bilbaíno Ángel Garma (1904-1993) fundador del psicoanálisis argentino. Bilbao: BBK. 2005.

MELIN, Goran.

Pragmatism and self-organization: research collaboration on the individual level. Research Policy, v.29, n.1, p.31-40. 2000.

MELIN,Goran; PERSSON, Olle.

HotelCosmopolitan: a bibliometric study of collaboration at some european universities. Journal of the American Society for Information Science, Los Angeles, v.49, n.1, p.43-48. 1998.

MERTON, Robert K.

Priorities in scientific discovery. American Sociological Review, Columbus, v.22, p.6-35. 1957.

MUÑOZ, Isabel.

La introducción del psicoanálisis en España. Revista de Historia de la Psicología, v.22, n.1, p.83-104. 2001.

OKUBO, Yuichi.

Bibliometrics indicators and analysis of research systems: methods and examples. Paris: Organisation for Economic Co-operation and Development (GD-97-41). 1997.

PEIRÓ, Jose María.

Colegios invisibles en psicología. In: Carpintero, Helio; Peiró, Jose María (Ed.). Psicología contemporánea: teoría y métodos cuantitativos para el estudio de la literatura científica. Valencia: Alfaplús. 1981.

PEÑARANDA ORTEGA, María.

La colaboración científica en la psicología social y de la personalidad: análisis bibliométrico del Journal of Personality and Social Psychology
(1965- 2000). Murcia: Ediciones Universidad de Murcia-PPU. 2004.

PÉREZ MATOS, Nuria.

La bibliografía, bibliometría y las ciencias afines. ACIMED, La Habana, v.10, n.3, p.176-195.

2002.

PRICE, Derek John de Solla.

Hacia una ciencia de la ciencia. Barcelona: Ariel. 1973.

PRICE, Derek John de Solla.

Policies of science?. Melbourne Journal of Politics, Melbourne, v.2, p.1-8. 1969.

PRICE, Derek John de Solla; BEAVER, Donald. Collaboration in an invisible college. American Psychologist, Washington, v.21, n.11, p.1011-1018. 1966.

PRPIC, Katarina.

Gender and productivity differentials in science. Scientometrics, New York, v.55, n.1, p.27-58. 2002.

PRPIC, Katarina.

Scientific fields and eminent scientists' productivity patterns and factors.

Scientometrics, New York, v.37, n.3, p.445-471. 1996.

QUIÑONES VIDAL, Elena; MUÑOZ JIMÉNEZ, Isabel.

Evolución de los conceptos psicoanalíticos en España (1922-1936): un análisis bibliométrico. Revista de Historia de la Psicología, Valencia, v.7, n.3, p.55-70. 1986.

QUIÑONES VIDAL, Elena; VERA FERRÁNDIZ, Juan Antonio.

La presencia de Freud en el psicoanálisis argentino actual (1974-1983). Revista de Historia de la Psicología, Valencia, v.9, n.4, p.357-368. 1988.

SANCHO, Rafael.

Indicadores bibliométricos utilizados en la evaluación de la ciencia y la Tecnología: revisión bibliográfica. Revista Española de Documentación Científica, Madrid, v.13, n.3-4, p.842-865. 1990.

SHAPIRO, Fred R.

Origins of bibliometrics, citation indexing, and citation analysis: the neglected legal literature. Journal of the American Society for Information Science, Los Angeles, v.43, n.5, p.337-339. 1992.

\section{SOON, Ang.}

A comment on the intellectual structures of information systems development. Accounting, Management and Information Technologies, Amsterdam, v.6, n.1-2, p.65-69. 1996. 
STACK, Steven

Gender and scholarly productivity: the case of criminal justice. Journal of Criminal Justice, v.30, n.3, p.175-182. 2002.

TVIRE, Paul; ERNO, Linda.

Exploring invisible scientific communities: studying networking relations within an educational research community - a finnish case. Higer Education, v.42, n.4, p.493-513. 2001.

VINKLER, Peter.

Evaluation of the publication activity of research teams by means of scientometric incicators. Current Science, Bangalore, v.79, n.5, p.325-334. 2000.

VIVAS, Jorge; URQUIJO, Sebastián. Análisis de redes aplicado a un estudio bibliométrico en psicología de la conducta.
IberPsicología, v. 4, n.1. Disponible en: http:// dialnet.unirioja.es/servlet/listaarticulos?tipo_ busqueda $=$ VOLUMEN\&revista_busqueda $=$ 2091\&clave_busqueda $=4.1999$.

ZAK DE GOLDSTEIN, Raquel.

Homenaje a Ángel Garma en ocasión de cumplir 70 años. Revista de Psicoanálisis, Buenos Aires, n.31, p.843-879. 1974.

ZALBIDEA, María Antonia; CANTÓN, Enrique; CARPINTERO, Helio.

La influencia española en la psicología de los países latinoamericanos: A. Garma y el

psicoanálisis en Argentina. Revista de Historia de la Psicología, Valencia, v.12, n.2, p.175-182. 1991.

ZUCKERMAN, Miron.

Scientific elite: Nobel laureates in the United States. London: Free Press. 1977.

\section{$\rightarrow \rightarrow \rightarrow<<<$}

